\title{
766.
}

\section{ON THE GEODESIC CURVATURE OF A CURVE ON A SURFACE.}

[From the Proceedings of the London Mathematical Society, vol. xII. (1881), pp. 110-117. Read April 14, 1881.]

THERE is contained in Liouville's Note II. to his edition of Monge's Application de l'Analyse à la Géométrie (Paris, 1850), see pp. 574 and 575, the following formula,

$$
\begin{aligned}
\frac{1}{\rho} & =-\frac{d i}{d s}+\frac{1}{2 G \sqrt{ } E} \frac{d G}{d u} \cos i-\frac{1}{2 E \sqrt{ } G} \frac{d E}{d v} \sin i \\
& =-\frac{d i}{d s}+\frac{\cos i}{\rho_{2}}+\frac{\sin i}{\rho_{1}}
\end{aligned}
$$

which gives the radius of geodesic curvature of a curve upon a surface when the position of a point on the surface is defined by the parameters $u, v$, belonging to a system of orthotomic curves; or, what is the same thing, such that

$$
d s^{2}=E d u^{2}+G d v^{2} .
$$

Writing with Gauss $p, q$ instead of $u, v$, I propose to obtain the corresponding formula in the general case where the parameters $p, q$ are such that

$$
d s^{2}=E d p^{2}+2 F d p d q+G d q^{2} .
$$

I call to mind that, if $P Q, P Q^{\prime}$ are equal infinitesimal arcs on the given curve and on its tangent geodesic, then the radius of geodesic curvature $\rho$ is, by definition, a length $\rho$ such that $2 \rho \cdot Q Q^{\prime}=\overline{P Q^{2}}$. More generally, if the curves on the surface are any two curves which touch each other, then $\rho$ as thus determined is the radius of relative curvature of the two curves. 
The notation is that of the Memoir, "Disquisitiones generales circa superficies curvas" (1827), Gauss, Werke, t. III.; see also my paper "On geodesic lines, in particular those of a quadric surface," Proc. Lond. Math. Society, t. IV. (1872), pp. 191-211, [508]; and Salmon's Solid Geometry, 3rd ed., 1874, pp. 251 et seq. The coordinates $(x, y, z)$ of a point on the surface are taken to be functions of two independent parameters $p, q$; and we then write

$$
\begin{gathered}
d x+\frac{1}{2} d^{2} x=a d p+a^{\prime} d q+\frac{1}{2}\left(\alpha d p^{2}+2 \alpha^{\prime} d p d q+\alpha^{\prime \prime} d q^{2}\right), \\
d y+\frac{1}{2} d^{2} y=b d p+b^{\prime} d q+\frac{1}{2}\left(\beta d p^{2}+2 \beta^{\prime} d p d q+\beta^{\prime \prime} d q^{2}\right), \\
d z+\frac{1}{2} d^{2} z=c d p+c^{\prime} d q+\frac{1}{2}\left(\gamma d p^{2}+2 \gamma^{\prime} d p d q+\gamma^{\prime \prime} d q^{2}\right): \\
E, F, G=a^{2}+b^{2}+c^{2}, \quad a a^{\prime}+b b^{\prime}+c c^{\prime}, \quad a^{\prime 2}+b^{\prime 2}+c^{\prime 2} ; \quad V^{2}=E G-F^{2} ;
\end{gathered}
$$

and therefore

$$
d s^{2}=E d p^{2}+2 F d p d q+G d q^{2}
$$

where $E, F, G$ are regarded as given functions of $p$ and $q$.

To determine a curve on the surface, we establish a relation between the two parameters $p, q$, or, what is the same thing, take $p, q$ to be functions of a single parameter $\theta$; and we write as usual $p^{\prime}, p^{\prime \prime}, q^{\prime}$, etc., to denote the differential coefficients of $p, q$, etc., in regard to $\theta$; we write also $E_{1}, E_{2}$, etc., to denote the differential coefficients $\frac{d E}{d p}, \frac{d E}{d q}$, etc. In the first instance, $\theta$ is taken to be an arbitrary parameter, but we afterwards take it to be the length $s$ of the curve from a fixed point thereof.

First formula for the radius of relative curvature.

Consider any two curves touching at the point $P$, coordinates $(x, y, z)$ which are regarded as given functions of $(p, q)$; where $(p, q)$ are for the one curve given functions, and for the other curve other given functions, of $\theta$.

The coordinates of a consecutive point for the one curve are then

where

$$
x+d x+\frac{1}{2} d^{2} x, \quad y+d y+\frac{1}{2} d^{2} y, \quad z+d z+\frac{1}{2} d^{2} z,
$$

$$
d p=p^{\prime} d \theta+\frac{1}{2} p^{\prime \prime} d \theta^{2}, \quad d q=q^{\prime} d \theta+\frac{1}{2} q^{\prime \prime} d \theta^{2} ;
$$

hence these coordinates are

$$
x+\left(a p^{\prime}+a^{\prime} q^{\prime}\right) d \theta+\frac{1}{2}\left(\alpha p^{\prime 2}+2 \alpha^{\prime} p^{\prime} q^{\prime}+\alpha^{\prime \prime} q^{\prime 2}\right) d \theta^{2}+\frac{1}{2}\left(a p^{\prime \prime}+\alpha^{\prime} q^{\prime \prime}\right) d \theta^{2}
$$

and for the other curve they are in like manner

$$
x+\left(a p^{\prime}+a^{\prime} q^{\prime}\right) d \theta+\frac{1}{2}\left(\alpha p^{\prime 2}+2 \alpha^{\prime} p^{\prime} q^{\prime}+\alpha^{\prime \prime} q^{\prime 2}\right) d \theta^{2}+\frac{1}{2}\left(a P^{\prime \prime}+a^{\prime} Q^{\prime \prime}\right) d \theta^{2},
$$


the only difference being in the terms which contain the second differential coefficients, $p^{\prime \prime}, q^{\prime \prime}$ for the first curve, and $P^{\prime \prime}, Q^{\prime \prime}$ for the second curve. Hence the differences of the coordinates are

$$
\begin{gathered}
\frac{1}{2}\left\{a\left(p^{\prime \prime}-P^{\prime \prime}\right)+a^{\prime}\left(q^{\prime \prime}-Q^{\prime \prime}\right)\right\} d \theta^{2}, \quad \frac{1}{2}\left\{b\left(p^{\prime \prime}-P^{\prime \prime}\right)+b^{\prime}\left(q^{\prime \prime}-Q^{\prime \prime}\right)\right\} d \theta^{2}, \\
\frac{1}{2}\left\{c\left(p^{\prime \prime}-P^{\prime \prime}\right)+c^{\prime}\left(q^{\prime \prime}-Q^{\prime \prime}\right)\right\} d \theta^{2},
\end{gathered}
$$

and consequently the distance $Q Q^{\prime}$ of the two consecutive points $Q, Q^{\prime}$ is

$$
=\frac{1}{2} \sqrt{\left(E, F, G \gamma p^{\prime \prime}-P^{\prime \prime}, q^{\prime \prime}-Q^{\prime \prime}\right)^{2}} d \theta^{2} .
$$

The squared are $\overline{P Q}^{2}$ is

$$
=\left(E, F, G \gamma p^{\prime}, q^{\prime}\right)^{2} d \theta^{2} ;
$$

and hence, if as before $2 \rho \cdot Q Q^{\prime}=\overline{P Q}^{2}$, that is, $\frac{1}{\rho}=2 Q Q^{\prime} \div \overline{P Q}^{2}$, then

$$
\frac{1}{\rho}=\frac{\sqrt{\left(E, F, G \gamma p^{\prime \prime}-P^{\prime \prime}, q^{\prime \prime}-Q^{\prime \prime}\right)^{2}}}{\left(E, F, G \gamma p^{\prime}, q^{\prime}\right)^{2}},
$$

the required formula for $\rho$.

Second formula for the radius of relative curvature.

We now take the variable $\theta$ to be the length $s$ of the curve measured from a fixed point thereof, so that $p^{\prime}, p^{\prime \prime}$, etc. denote $\frac{d p}{d s}, \frac{d^{2} p}{d s^{2}}$, etc. We have therefore

and the formula becomes

$$
1=\left(E, F, G \gamma p^{\prime}, q^{\prime}\right)^{2},
$$

$$
\frac{1}{\rho}=\sqrt{\left(E, F, G^{\prime} \chi p^{\prime \prime}-P^{\prime \prime}, q^{\prime \prime}-Q^{\prime \prime}\right)^{2}} .
$$

But, differentiating the above equation as regards the curve, we find

$$
0=2\left(E, F, G \gamma p^{\prime}, q^{\prime} \gamma p^{\prime \prime}, q^{\prime \prime}\right)+\left(\dot{E}, \dot{F}, \dot{G} \gamma p^{\prime}, q^{\prime}\right)^{2},
$$

where $\dot{E}, \dot{F}, \dot{G}$ are used to denote the complete differential coefficients $E_{1} p^{\prime}+E_{2} q^{\prime}$, etc. And similarly, differentiating in regard to the tangent geodesic, we obtain

$$
0=2\left(E, F, G \gamma p^{\prime}, q^{\prime} \chi P^{\prime \prime}, Q^{\prime \prime}\right)+\left(\dot{E}, \dot{F}, \dot{G} \gamma\left(p^{\prime}, q^{\prime}\right)^{2} ;\right.
$$

and hence, taking the difference of the two equations,

$$
0=\left(E, F, G \gamma p^{\prime}, q^{\prime} \chi p^{\prime \prime}-P^{\prime \prime}, q^{\prime \prime}-Q^{\prime \prime}\right) \text {. }
$$

Hence, in the equation for $\frac{1}{\rho}$, the function under the radical sign may be written $\left(E, F, G \gamma p^{\prime}, q^{\prime}\right)^{2} \cdot\left(E, F, G \gamma p^{\prime \prime}-P^{\prime \prime}, q^{\prime \prime}-Q^{\prime \prime}\right)^{2}-\left\{\left(E, F, G \gamma p^{\prime}, q^{\prime} \chi p^{\prime \prime}-P^{\prime \prime}, q^{\prime \prime}-Q^{\prime \prime}\right)\right\}^{2}$, 
which is identically

$$
=\left(E G-F^{2}\right)\left\{p^{\prime}\left(q^{\prime \prime}-Q^{\prime \prime}\right)-q^{\prime}\left(p^{\prime \prime}-P^{\prime \prime}\right)\right\}^{2} .
$$

Hence, extracting the square root, and for $\sqrt{E^{\prime} G-F^{2}}$ writing $V$, we have

or say

$$
\frac{1}{\rho}=V\left\{p^{\prime}\left(q^{\prime \prime}-Q^{\prime \prime}\right)-q^{\prime}\left(p^{\prime \prime}-P^{\prime \prime}\right)\right\}
$$

$$
\frac{1}{\rho}=V\left(p^{\prime} q^{\prime \prime}-q^{\prime} p^{\prime \prime}\right)-V\left(p^{\prime} Q^{\prime \prime}-q^{\prime} P^{\prime \prime}\right),
$$

which is the new formula for the radius of relative curvature.

Formula for the radius of geodesic curvature.

In the paper "On Geodesic Lines, etc." p. 195, [vol. viII. of this Collection, p. 160], writing $E G-F^{2}=V^{2}$, and $P^{\prime \prime}, Q^{\prime \prime}$ in place of $p^{\prime \prime}, q^{\prime \prime}$, the differential equation of the geodesic line is obtained in the form

$$
\begin{aligned}
\left(E p^{\prime}+F q^{\prime}\right)\left\{\left(2 F_{1}-E_{2}\right) p^{\prime 2}+2 G_{1} p^{\prime} q^{\prime}+G_{2} q^{\prime 2}\right\} & -\left(F p^{\prime}+G q^{\prime}\right)\left\{E_{1} p^{\prime 2}+2 E_{2} p^{\prime} q^{\prime}+\left(2 F_{2}-G_{1}\right) q^{\prime 2}\right\} \\
& +2 V^{2}\left(p^{\prime} Q^{\prime \prime}-q^{\prime} P^{\prime \prime}\right)=0 ;
\end{aligned}
$$

or, denoting by $\Omega$ the first two lines of this equation, we have

$$
V\left(p^{\prime} Q^{\prime \prime}-q^{\prime} P^{\prime \prime}\right)=-\frac{\frac{1}{2}}{V} \Omega \text {. }
$$

The foregoing equation gives therefore, for the radius of geodesic curvature,

$$
\frac{1}{\rho}=V\left(p^{\prime} q^{\prime \prime}-p^{\prime \prime} q^{\prime}\right)+\frac{\frac{1}{2}}{V} \Omega,
$$

which is an expression depending only upon $p^{\prime}, q^{\prime}$, the first differential coefficients (common to the curve and geodesic), and on $p^{\prime \prime}, q^{\prime \prime}$, the second differential coefficients belonging to the curve.

Observe that $\Omega$ is a cubic function of $p^{\prime}, q^{\prime}$ : we have

$$
\Omega=\left(\mathfrak{A}, \mathfrak{B}, \mathfrak{C}, \mathfrak{D} \mathcal{X} p^{\prime}, q^{\prime}\right)^{3},
$$

the values of the coefficients being

$$
\begin{aligned}
& \mathfrak{A}=2 E F_{1}-E E_{2}-F E_{1}, \\
& \mathfrak{B}=2 E G_{1}+2 F F_{1}-3 F E_{2}-G E_{1}, \\
& \mathfrak{C}=E G_{2}+3 F G_{1}-2 F F_{2}-2 G E_{2}, \\
& \mathfrak{D}=F G_{2}-2 G F_{2}+G G_{1} .
\end{aligned}
$$


The Special Curves, $p=$ constant and $q=$ constant.

Consider the curve $p=$ const. For this curve $p^{\prime}=0, p^{\prime \prime}=0$; therefore also $G q^{\prime 2}=1$, and, if $R$ be the radius of geodesic curvature, then

$$
\frac{1}{R}=\frac{\frac{1}{2}}{V} D q^{\prime 3}, \quad=\frac{\frac{1}{2}}{V} \frac{D}{G \sqrt{ } G} .
$$

Similarly for the curve $q=$ const. Here $q^{\prime}=0, q^{\prime \prime}=0$; therefore $E p^{\prime 2}=1$, and, if $S$ be the radius of geodesic curvature, then

$$
\frac{1}{S}=\frac{\frac{1}{2}}{V} \mathfrak{A} p^{\prime 3}, \quad=\frac{\frac{1}{2}}{V} \frac{\mathfrak{A}}{E \sqrt{ } E},
$$

These values of $R$ and $S$ are interesting for their own sakes, and they will be introduced into the expression for the radius of geodesic curvature $\rho$ of the general curve.

Transformed Formula for the Radius of Geodesic Curvature.

From the values of $\frac{1}{R}, \frac{1}{S}$, we have

$$
\frac{1}{\rho}-\frac{q^{\prime} \sqrt{ } G}{R}-\frac{p^{\prime} \sqrt{ } E}{S}=V\left(p^{\prime} q^{\prime \prime}-p^{\prime \prime} q^{\prime}\right)+\frac{\frac{1}{2}}{V}\left\{\Omega-\frac{\mathfrak{A}}{E} p^{\prime}-\frac{\mathfrak{D}}{G} q^{\prime}\right\}
$$

where the term in \{\} is

$$
=\mathfrak{A} p^{\prime 3}-\frac{\mathfrak{U}}{E} p^{\prime}+\mathfrak{B} p^{\prime 2} q^{\prime}+\mathfrak{C}^{\prime} p^{\prime} q^{\prime 2}+\mathfrak{D} q^{\prime 3}-\frac{\mathfrak{D}}{G} q^{\prime} .
$$

The terms in $\mathfrak{A}$ are

$$
=-\frac{\mathfrak{A}}{E} p^{\prime}\left(1-E p^{\prime 2}\right), \quad=-\frac{\mathfrak{A}}{E} p^{\prime}\left(2 F p^{\prime} q^{\prime}+G q^{\prime 2}\right),
$$

and those in $\mathfrak{D}$ are

$$
=-\frac{\mathfrak{D}}{G} q^{\prime}\left(1-G q^{\prime 2}\right), \quad=-\frac{\mathfrak{D}}{G} q^{\prime}\left(E p^{\prime 2}+2 F p^{\prime} q^{\prime}\right) .
$$

Hence the whole expression contains the factor $p^{\prime} q^{\prime}$, and is, in fact,

$$
=p^{\prime} q^{\prime}\left\{p^{\prime}\left(\mathfrak{B}-\frac{2 \mathfrak{A} F}{E}-\frac{D E E}{G}\right)+q^{\prime}\left(\mathfrak{B}-\frac{\mathfrak{A} G}{E}-\frac{2 D F}{G}\right)\right\} ;
$$

or substituting for $\mathfrak{A}, \mathfrak{B}, \mathfrak{C}, \mathfrak{D}$ their values, this is

$$
\begin{aligned}
=p^{\prime} q^{\prime}\{ & p^{\prime}\left(-G E_{1}+E G_{1}+\frac{2 F^{2} E_{1}}{E}-2 F F_{1}-F E_{2}+2 E F_{2}-\frac{E F G_{2}}{G}\right) \\
& \left.+q^{\prime}\left(-G E_{2}+E G_{2}-\frac{2 F^{2} G_{2}}{G}+2 F F_{2}+F G_{1}-2 G F_{1}+\frac{G F E_{1}}{E}\right)\right\},
\end{aligned}
$$


say this is

and the formula thus is

$$
=p^{\prime} q^{\prime}\left(L p^{\prime}+M q^{\prime}\right)
$$

$$
\frac{1}{\rho}-\frac{q^{\prime} \sqrt{ } G}{R}-\frac{p^{\prime} \sqrt{ } E}{S}=V\left(p^{\prime} q^{\prime \prime}-p^{\prime \prime} q^{\prime}\right)+\frac{\frac{1}{2}}{V} p^{\prime} q^{\prime}\left(L p^{\prime}+M q^{\prime}\right) .
$$

Taking $\phi, \theta$ to be the inclination of the curve to the curves $q=$ const., $p=$ const., respectively, and $\omega(=\phi+\theta)$ the inclination of these two curves to each other, then

$$
\begin{array}{lll}
\cos \phi=\frac{F p^{\prime}+G q^{\prime}}{\sqrt{ } G}, & \cos \theta=\frac{E p^{\prime}+F q^{\prime}}{\sqrt{ } E}, & \cos \omega=\frac{F}{\sqrt{E G}}, \\
\sin \phi=\frac{V p^{\prime}}{\sqrt{ } G}, & \sin \theta=\frac{V q^{\prime}}{\sqrt{ } E}, & \sin \omega=\frac{V}{\sqrt{ } E G} ;
\end{array}
$$

hence $\frac{\sin \phi}{\sin \omega}=p^{\prime} \sqrt{ } E, \frac{\sin \theta}{\sin \omega}=q^{\prime} \sqrt{ } G$, and the formula may also be written

$$
\frac{1}{\rho}-\frac{\sin \theta}{\sin \omega} \frac{1}{R}-\frac{\sin \phi}{\sin \omega} \frac{1}{S}=V\left(p^{\prime} q^{\prime \prime}-p^{\prime \prime} q^{\prime}\right)+\frac{\frac{1}{2}}{V} p^{\prime} q^{\prime}\left(L p^{\prime}+M q^{\prime}\right) \text {. }
$$

The Orthotomic Case $F=0$, or $d s^{2}=E d p^{2}+G d q^{2}$.

The formula becomes in this case much more simple. We have

$$
1=E p^{\prime 2}+G q^{\prime 2}, \quad V=\sqrt{E G}, \quad \omega=90^{\circ}, \quad \sin \theta=\cos \phi ;
$$

and the term $L p^{\prime}+M q^{\prime}$ becomes $=E \dot{G}-\dot{E} G$, if, as before, $\dot{E}, \dot{G}$ denote the complete differential coefficients $E_{1} p^{\prime}+E_{2} q^{\prime}$ and $G_{1} p^{\prime}+G_{2} q^{\prime}$. The formula then is

$$
\frac{1}{\rho}-\frac{\cos \phi}{R}-\frac{\sin \phi}{S}=V\left(p^{\prime} q^{\prime \prime}-p^{\prime \prime} q^{\prime}\right)+\frac{\frac{1}{2}}{V}(E \dot{G}-\dot{E} G),
$$

where the values $\frac{1}{R}$ and $\frac{1}{S}$ are now $=\frac{\frac{1}{2} G_{1}}{G \sqrt{ } E}$ and $\frac{-\frac{1}{2} E_{2}}{E \sqrt{ } G}$, respectively. But we have moreover $\phi=\tan ^{-1} \frac{p^{\prime} \sqrt{ } E}{q^{\prime} \sqrt{ } G}$, and thence

$$
\begin{aligned}
\phi^{\prime} & =q^{\prime} \sqrt{ } G\left(p^{\prime \prime} \sqrt{ } E+\frac{\frac{1}{2} p^{\prime} \dot{E}}{\sqrt{ } E}\right)-p^{\prime} \sqrt{ } E\left(q^{\prime \prime} \sqrt{ } G+\frac{\frac{1}{2} q^{\prime} \dot{G}}{\sqrt{ } G}\right) \\
& =-V\left(p^{\prime} q^{\prime \prime}-p^{\prime \prime} q^{\prime}\right)-\frac{\frac{1}{2}}{V} p^{\prime} q^{\prime}(E \dot{G}-\dot{E} G)
\end{aligned}
$$

or the formula finally is

$$
\frac{1}{\rho}-\frac{\cos \phi}{R}-\frac{\sin \phi}{S}+\phi^{\prime}=0
$$

which is Liouville's formula referred to at the beginning of the present paper. It will be recollected that $\phi^{\prime}$ is the differential coefficient $\frac{d \phi}{d s}$ with respect to the arc $s$ of the curve. 
AdDITION.-Since the foregoing paper was written, I have succeeded in obtaining a like interpretation of the term

$$
V\left(p^{\prime} q^{\prime \prime}-p^{\prime \prime} q^{\prime}\right)+\frac{\frac{1}{2}}{V} p^{\prime} q^{\prime}\left(L p^{\prime}+M q^{\prime}\right)
$$

which belongs to the general case. I find that these terms are, in fact, $=-\dot{\phi}+\omega_{1} p^{\prime}$; or, what is the same thing (since $\omega=\phi+\theta$ and therefore $\omega_{1} p^{\prime}+\omega_{2} q^{\prime}=\dot{\phi}+\dot{\theta}$ ), are $=\dot{\theta}-\omega_{2} q^{\prime}$. It will be recollected that $\phi$ is the inclination of the curve to the curve $q=c$, which passes through a given point of the curve, $\dot{\phi}$ is the variation of $\phi$ corresponding to the passage to the consecutive point of the curve, viz., $\phi+\dot{\phi} d s$ is the inclination at this consecutive point to the curve $q=c+d c$, which passes through the consecutive point; $\omega$ is the inclination to each other of the curves $p=b, q=c$, which pass through the given point of the curve, $\omega_{1}$ the variation corresponding to the passage along the curve $q=c$, viz., $\omega+\omega_{1} d s$ is the inclination to each other of the curves $p=b+d b, q=c$; and the like as regards $\dot{\theta}$ and $\omega_{2}$.

For the demonstration, we have, as above,

where

$$
\phi=\tan ^{-1} \frac{V p^{\prime}}{F p^{\prime}+G q^{\prime}}, \quad \omega=\tan ^{-1} \frac{V}{F^{\prime}},
$$

$$
V=\sqrt{E G-F^{2}}
$$

and moreover $E p^{\prime 2}+2 F p^{\prime} q^{\prime}+G q^{\prime 2}=1$. In virtue of this last equation,

and we have

$$
V^{2} p^{\prime 2}+\left(F p^{\prime}+G q^{\prime}\right)^{2}=G ;
$$

where

$$
\dot{\phi}=-V\left(p^{\prime} q^{\prime \prime}-p^{\prime \prime} q^{\prime}\right)+\frac{1}{G} \square,
$$

$$
\square=\left(F p^{\prime}+G q^{\prime}\right) p^{\prime} \dot{V}-V p^{\prime}\left(\dot{F} p^{\prime}+\dot{G} q^{\prime}\right) ;
$$

or, since $V^{2}=E G-F^{2}$, and thence $2 V \dot{V}=G \dot{E}-2 F \dot{F}+E \dot{G}$, we have

$$
\square=\frac{\frac{1}{2} p^{\prime}}{V}\left\{\left(F p^{\prime}+G q^{\prime}\right)\left(G \dot{E}-2 F^{\prime} \dot{F}+E \dot{G}\right)-2\left(E G-F^{2}\right)\left(\dot{F} p^{\prime}+\dot{G} q^{\prime}\right)\right\} .
$$

Substituting herein for $\dot{E}, \dot{F}, \dot{G}$ their values $E_{1} p^{\prime}+E_{2} q^{\prime}, \quad F_{1} p^{\prime}+F_{2} q^{\prime}, G_{1} p^{\prime}+G_{2} q^{\prime}$, the term in \{\} becomes

where

$$
=I p^{\prime 2}+J p^{\prime} q^{\prime}+K q^{\prime 2}
$$

$$
\begin{aligned}
& I=F G E_{1}-2 E G F_{1}+E F G_{1}, \\
& J=G^{2} E_{1}-2 F G F_{1}+\left(-E G+2 F^{2}\right) G_{1}+F G E_{2}-2 E G F_{2}+E F G_{2}, \\
& K=G^{2} E_{2}-2 F G F_{2}+\left(-E G+2 F^{2}\right) G_{2} .
\end{aligned}
$$

But from the equation $\omega=\tan ^{-1} \frac{V}{F}$, differentiating in regard to $p$, we obtain

$$
\omega_{1}=\frac{\frac{1}{2}}{E G V}(F G \dot{E}-2 E G \dot{F}+E F \dot{G})=\frac{\frac{1}{2}}{E G V} I
$$

C. XI. 
or, for $p$ writing

$$
p^{\prime}\left(E p^{\prime 2}+2 F p^{\prime} q^{\prime}+G q^{\prime 2}\right), \quad=E p^{\prime}\left(p^{\prime 2}+2 \frac{F}{E} p^{\prime} q^{\prime}+\frac{G}{E} q^{\prime 2}\right)
$$

we have

$$
\begin{aligned}
\dot{\phi}-\omega_{1} p^{\prime}=-V\left(p^{\prime} q^{\prime \prime}-p^{\prime \prime} q^{\prime}\right) & +\frac{\frac{1}{2} p^{\prime}}{G V}\left(I p^{\prime 2}+J p^{\prime} q^{\prime}+K q^{\prime 2}\right) \\
& -\frac{\frac{1}{2} p^{\prime}}{G V} I\left(p^{\prime 2}+2 \frac{F}{E} p^{\prime} q^{\prime}+\frac{G}{E} q^{\prime 2}\right) .
\end{aligned}
$$

The terms in $p^{\prime 3}$ destroy each other, and the form thus is

$$
\dot{\phi}-\omega_{1} p^{\prime}=-V\left(p^{\prime} q^{\prime \prime}-p^{\prime \prime} q^{\prime}\right)-\frac{\frac{1}{2}}{V} p^{\prime} q^{\prime}\left(L p^{\prime}+M q^{\prime}\right)
$$

where

$$
\begin{aligned}
& L=-\frac{J}{G}+\frac{2 I F}{G E}, \\
& M=-\frac{K}{G}+\frac{I}{E} ;
\end{aligned}
$$

and, upon substituting herein for $I, J, K$ their values, we find

$$
\begin{aligned}
& L=-G E_{1}+E G_{1}+\frac{2 F^{2} E_{1}}{F}-2 F F_{1}-F E_{2}+2 E F_{2}-\frac{E F G_{2}}{G}, \\
& M=-G E_{2}+E G_{2}-\frac{2 F^{2} G_{2}}{G}+2 F F_{2}+F G_{1}-2 G F_{1}+\frac{G F E_{1}}{E}
\end{aligned}
$$

viz., these are the values denoted above by the same letters $L, M$. The final result thus is

$$
\begin{aligned}
\frac{1}{\rho}-\frac{q^{\prime} \sqrt{ } G}{R}-\frac{p^{\prime} \sqrt{ } E}{S} & =-\dot{\phi}+\omega_{1} p^{\prime}, \\
& =\dot{\theta}-\omega_{2} q^{\prime},
\end{aligned}
$$

where the meanings of the symbols have been already explained. A formula substantially equivalent to this, but in a different (and scarcely properly explained) notation, is given, Aoust, "Théorie des coordonnées curvilignes quelconques," Annali di Matem., t. II. (1868), pp. 39-64; and I was, in fact, led thereby to the foregoing further investigation.

As to the definition of the radius of geodesic curvature, I remark that, for a curve on a given surface, if $P Q$ be an infinitesimal are of the curve, then if from $Q$ we let fall the perpendicular $Q M$ on the tangent plane at $P$ (the point $M$ being thus a point on the tangent $P T$ of the curve), and if from $M$, in the tangent plane and at right angles to the tangent, we draw $M N$ to meet the osculating plane of the curve in $N$, then $M N$ is in fact equal to the infinitesimal arc $Q Q^{\prime}$ mentioned near the beginning of the present paper, and the radius of geodesic curvature $\rho$ is thus a length such that $2 \rho \cdot M N=\overline{P Q}^{2}$. 